\title{
From 1 Sun to 10 Suns c-Si Cells by Optimizing Metal Grid, Metal Resistance, and Junction Depth
}

\author{
Vikrant A. Chaudhari and Chetan S. Solanki \\ Department of Energy Science and Engineering, Indian Institute of Technology Bombay, Powai Mumbai 400076, India \\ Correspondence should be addressed to Vikrant A. Chaudhari, vikrant.c@iitb.ac.in
}

Received 6 March 2009; Accepted 21 July 2009

Recommended by Wayne A. Anderson

\begin{abstract}
Use of a solar cell in concentrator PV technology requires reduction in its series resistance in order to minimize the resistive power losses. The present paper discusses a methodology of reducing the series resistance of a commercial c-Si solar cell for concentrator applications, in the range of 2 to 10 suns. Step by step optimization of commercial cell in terms of grid geometry, junction depth, and electroplating of the front metal contacts is proposed. A model of resistance network of solar cell is developed and used for the optimization. Efficiency of unoptimized commercial cell at 10 suns drops by $30 \%$ of its 1 sun value corresponding to resistive power loss of about $42 \%$. The optimized cell with grid optimization, junction optimization, electroplating, and junction optimized with electroplated contacts cell gives resistive power loss of $20 \%, 16 \%, 11 \%$, and $8 \%$, respectively. An efficiency gain of $3 \%$ at 10 suns for fully optimized cell is estimated.
\end{abstract}

Copyright ( 2009 V. A. Chaudhari and C. S. Solanki. This is an open access article distributed under the Creative Commons Attribution License, which permits unrestricted use, distribution, and reproduction in any medium, provided the original work is properly cited.

\section{Introduction}

Solar PV technology is gaining importance as one of the major alternative source of energy. This is evident from the increase in solar cell production and demand during the past years [1]. Amongst the various PV technologies, Si is one of the widely used semiconductors for the fabrication of solar cells. About $80 \%$ to $90 \%$ of the PV cells manufactured worldwide is Si wafer based, which could be either crystalline $\mathrm{Si}$ (c-Si) or multicrystalline $\mathrm{Si}(\mathrm{mc}-\mathrm{Si})$, and this trend is to remain nearly same in the coming years $[2,3]$. Even though there is an increase in demand of the solar cell production the price of power generated from the solar cells has not changed over the years and is quite high at about $4-5 \$ / \mathrm{W}_{\mathrm{p}}$ [4]. Due to this the price of electricity generated from the solar cells is also high when compared with the conventional electricity price.

In case of c-Si modules about $50 \%$ is contributed by the base material, $\mathrm{Si}$ [5]. Efforts are in progress to reduce the material usage in the solar cells. This includes use of thin c-Si wafers $[6,7], \mathrm{Si}$ in the form of ribbon $[8,9]$, thin film c-Si on glass cells $[10,11]$, and concentrator c-Si cells $[12,13]$.
Concentrator solar cell technologies are being developed for the concentration ratio, $(C R)$ or $X$ (also referred as suns) of as low as $2[14,15]$ to as high as $1000[16,17]$. For high concentration ratio ( $>100$ suns) cells based on III$\mathrm{V}$ materials are suited best while for the low to medium concentration level (2 to 100 suns) c-Si-based solar cells can be used $[18,19]$.

The low-cost potential of concentrator solar cells is due to reduction in cell area for a given power output. Cell area decreases as inverse of the concentration ratio. Due to this inverse relationship, reduction in cell area is about $90 \%$ of the 1-sun cell area for concentration ratio of 10 suns. Further increasing the concentration ratio does not result in significant cell area reduction, and hence it does not result in further significant cost reduction when the solar cell efficiencies are assumed to be low, in range of 14 to $16 \%$, as in case of commercially available c-Si cells. The advantage of working with low concentration ratio c-Si cell technology ( 2 to 10 suns) is that the processes which are used currently in industry can be used to fabricate solar cells suited for concentration. Another advantage of lowconcentrator PV systems is that it offers higher tolerance 
for sun-tracking, both in terms of tracking accuracy and tracking infrastructure [20]. Therefore low concentration cSi cell technology has the potential to reduce the cost/ $\mathrm{W}_{\mathrm{p}}$ generated from the solar cells.

One of the major issues for the solar cell to operate at concentration levels is its series resistance, $R_{s}$. The series resistance causes the $I^{2} R_{s}$ resistive power loss in the solar cells and thus reduces its performance by reducing the $F F$ of solar cells. The expression for solar cell efficiency $\eta$, as a function of concentration ratio, is given in (1).

$$
\eta=\frac{\left(J_{\mathrm{sc}} * C R\right) *(k T / q) \ln \left(\left(\left(J_{\mathrm{sc}} * C R\right) / J_{0}\right)+1\right) * F F(C R)}{C R * P_{\mathrm{in}}} .
$$

Here $J_{\text {sc }}$ is short circuit current density, $P_{\text {in }}$ in incident power density corresponding to $\mathrm{AM} 1.5, J_{o}$ is reverse saturation current density, $q$ is elemental charge, and $F F(C R)[21]$ is $F F$ as a function of concentration ratio. As per (1) the efficiency of solar cell increases with concentration ratio provided that $F F(C R)$ is constant. Typically, when a solar cell, designed for 1-sun application, is used at higher concentration its efficiency decreases with increase in concentration ratio due to decrease in FF. A solar cell operates at maximum efficiency when its ohmic voltage drop becomes equal to thermal voltage $(k T / q)$ [22]. For a cell working under light concentration, this can be written as

$$
J_{\mathrm{sc}}(1 \operatorname{sun}) * C R * R_{s}=\frac{k T}{q} .
$$

Equation (2) suggests that in order to avoid the cell efficiency drop under concentration, the cell's $R_{s}$ should vary as inverse of concentration ratio. Thus in order to use a cell at 10 suns its $R_{s}$ should be reduced by a factor of 10 . The series resistance in the solar cells is contributed by the bulk region, emitter region, metal grid consisting of the metal fingers and bus-bars, and the contact resistance between the metal and semiconductor. The contribution of the top region, metal grid, and emitter is about $90 \%-95 \%$ of the total series resistance of a solar cell $[23,24]$. Thus by optimizing the emitter and metal region of a cell, significant reduction in $R_{s}$ can be obtained.

The present paper discusses an analytical approach for designing a c-Si concentrator solar cell for 10 suns application. It is shown that the current industrial solar cell fabrication setup which is used for 1 sun cell fabrication can also be used for 10 suns cell fabrication. Design of solar cell is presented in steps of grid geometry optimization, junction depth optimization and electroplated metal contact, and finally combination of these. Reduction in series resistance at various stages of cell design fabrication is analysed, and effect of individual design steps on solar cell performance is presented.

\section{Series Resistance Model of a Solar Cell}

The current in a solar cell flows vertically in the base, horizontally in the emitter layer, then through the fingers and bus bars. During the flow of the current the solar cell has to overcome various resistances, in the bulk, emitter, and the metal grid region. Most of the resistive losses occur in the top region of the solar cell especially in the thin emitter region and in the metal fingers. In order to estimate the series resistance of a cell under concentration, a simplified resistive network is derived based on the resistance model given by Handy [25].

Since current flow is one-dimensional, the current generated in the emitter region is assumed to be collected at the fingers and then transported to the bus-bar; hence no direct current flow is considered from the emitter to busbar. This eliminates the contact resistance between the busbar and the emitter and the emitter resistance between the semiconductor and the bus-bar; thus making the resistive model simplified. Power loss equations are considered for calculating the resistance in the emitter layer and metal grid fingers. Contact resistance between the metal and semiconductor is calculated from the contact resistivity information available from literature.

A solar cell of length, $L$, width, $W$, with thickness, $t$, of about $280 \mu \mathrm{m}$ is considered for the evaluation of the resistance model. The cell structure considered has the busbar running along length in the middle with fingers on either side. A resistance network is shown in Figure 1. Since current is collected from either side of the bus-bar and also from either side of fingers from emitter region, there are two emitter resistances in parallel and two finger resistances, $\left(R_{m}=R_{f}+R_{f c}\right)$ in parallel. Since the current flows from both the side of the fingers, two parallel resistance branches are considered and divided by two as shown in Figure 1. The total emitter resistance depends on the number of fingers, $n$, as depicted in Figure 1. The overall series resistance of a cell is obtained by solving the resistance network of Figure 1, and the equivalent series resistance obtained is given in (3). A detailed derivation of the calculation of series resistance is given in the appendix:

$$
R_{s}=R_{\mathrm{bus}}+R_{\mathrm{bulk}}+\frac{R_{\mathrm{emitter}}}{2(n+1)}+f\left(n,\left(R_{f}+R_{f c}\right), R_{\mathrm{bus}}\right) .
$$

\section{Case-1: Grid Optimization}

Grid optimization is an important exercise for keeping the resistive losses and shading losses to a minimum level. It is more important for concentrator cell application as in this cases that the generated current levels are higher than 1 sun levels which results in higher resistive losses, that is, higher $I^{2} R_{s}$ losses. Most grid design methodologies that are published in literature are for the 1 sun solar cells [26]. The grid design methodology for concentrator cells and especially for the low levels of concentrators is rarely published. The present study provides the design methodology of front contact grid geometry for a given concentration ratio.

The size and shape of the front contact grid is tradeoff between the shading and the resistive power loss. More number of lines or wide fingers would reduce the resistance but at the same time will cause shading that will reduce the short-circuit current. Less numbers of fingers would result in 


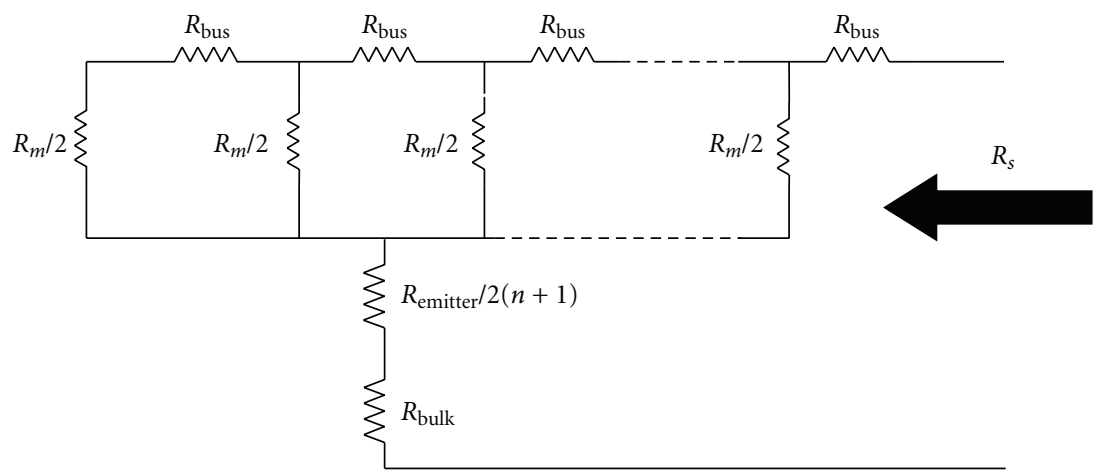

FIGURE 1: Resistance network of the solar cells $\left(R_{\text {bus }}\right.$ Bus-bar resistance, $R_{m}$ metal line resistance consisting of metal finger resistance, $R_{f}$ and metal finger to semiconductor contact resistance, $R_{f c}, R_{\text {emitter }}$ Emitter resistance, $R_{\text {bulk }}$ Bulk resistance).

increased short-circuit current and higher resistive loss in the emitter. Thus the number of fingers and width of fingers and bus-bars need to be optimized for a given area and power loss.

A model of resistance network explained in Section 2 is used for grid optimization. The resistive network is solved, and a lumped value of series resistance is calculated for the given solar cell. The value of the series resistance is then used in the I-V curve equation to obtain the I-V and P-V curves. The equation governing the I-V and P-V curve is as follows:

$$
\begin{aligned}
I_{L} & =I_{\mathrm{sc}}-I_{o} *\left(\exp ^{\left(V_{L}+I_{L} * R_{s}\right) / v_{t}}-1\right), \\
P_{L} & =V_{L} * I_{L}
\end{aligned}
$$

where $I_{L}, V_{L}$, and $P_{L}$ are the load current, load voltage, and the load power respectively, $I_{o}$ is the reverse saturation current derived from $J_{o}$, and $v_{t}=k T / q$ is the thermal voltage.

From (4) we plot the I-V and P-V curves of the solar cell and then calculate the peak power, $\left(P_{\max }\right)$. This $P_{\max }$ depends on the series resistance, which is a function of grid geometry consisting of the number of fingers, $n$ the finger width, $w_{f}$ bus-bar width, $w_{b}$ the emitter sheet resistance, $r_{s}$ metal line sheet resistance, and $r_{m}$ the concentration ratio. All these parameters are taken into consideration for calculating the $R_{s}$ (refer to appendix).

The grid optimization is carried out while varying only two parameters: the number of fingers, $n$, and the bus-bar width $w_{b}$. Other parameters like cell length, $L$, width, $W$, finger width, $w_{f}$, sheet resistance of emitter, $r_{s}$, and sheet resistance of metal fingers and bus-bar, $r_{m}$, are kept constant as there are practical limitations on those parameters. The description of the process is explained in the flow chart of Figure 2. The initial parameters with which the grid optimization starts are referred as the parameters of unoptimized concentrator cell or 1 sun cell. These are noted in Table 1.

The flowchart begins with input parameters of unoptimized concentrator solar cell (Table 1). At first the value of series resistance is calculated for initial value of $n=1$, and this value of series resistances is then used in the I-V and P-V curve equation to obtain the $P_{\max }$. Then $n$ is incremented and $P_{\max }$ is calculated again, the loop repeat itself till $n$ reaches 100 (predefined number of fingers), and corresponding value
TABLE 1: Geometrical parameters used in the simulation.

\begin{tabular}{lcc}
\hline$L$ (length) & 4 & $\mathrm{~cm}$ \\
$W$ (width) & 4 & $\mathrm{~cm}$ \\
$r_{s}$ (sheet resistance of emitter) & 45 & $\Omega / \mathrm{square}$ \\
$r_{m}$ (sheet resistance of metal fingers and bus-bars) & 3 & $\mathrm{~m} \Omega / \mathrm{square}$ \\
$w_{f}$ (width of fingers) & 150 & $\mu \mathrm{m}$ \\
$w_{b}$ (width of bus-bar) & 2 & $\mathrm{~mm}$ \\
$n$ (number of fingers) & 11 & $\mathrm{Nil}$ \\
$l_{f}$ (length of fingers) & 3.7 & $\mathrm{~cm}$ \\
$s$ (spacing between fingers) & 3.5 & $\mathrm{~mm}$ \\
$\rho_{c}$ (contact resistivity) & 0.1 & $\mathrm{~m} \Omega \mathrm{cm}^{2}$ \\
\hline
\end{tabular}

of $P_{\max }$ is noted as shown in flow chart of Figure 2. From the $P_{\max }$ data obtained for different values of $n$, a maximum value of $P_{\max }$, that is, $\left(P_{1}=P_{\max }(\max )\right)$ is obtained as shown in Figure 3 . The value of $n$, corresponding to maximum power value $\left(P_{1}\right)$, is used in loop B (see Figure 2) for further power maximization, which is done by optimizing busbar width, $w_{b}$. The procedure remains similar to that of maximizing power for given number of fingers. The loop $\mathrm{B}$ starts with initial guess value of the $w_{b}$, which is set to $0.5 \mathrm{~mm}$. For every value of $w_{b}$, corresponding values of $R_{s}$ and $P_{\max }$ are calculated from the $\mathrm{I}-\mathrm{V}$ and $\mathrm{P}-\mathrm{V}$ curves. This calculation continues till all the possible combinations of $w_{b}$ are used $\left(w_{b} \leq 2 \mathrm{~mm}\right)$ and the maximized value of power of loop $\left.\mathrm{B}, P_{2}=P_{\max }(\max )\right)$ is noted as shown in Figure 3. The range of $n$ and $w_{b}$ is defined based on allowable resistive and shadowing losses; normally it should be less than $10 \%$ of overall power generated.

Maximized values for $P_{\max }\left(P_{1}\right.$ and $\left.P_{2}\right)$ for $n$ and $w_{b}$ obtained from loop A and loop B are compared for equality. If the $P_{2}$ is different from $P_{1}$, the loop A and loop B (Figure 2) repeat themselves till $P_{1}$ becomes equal to $P_{2}$. It is found that the values of $P_{1}, P_{2}$ go on increasing with the number of iterations and then stabilize for a particular combination of grid geometry parameters. If the condition of $P_{1}=P_{2}$ is obtained, then the loop is terminated. The condition of $P_{1}=P_{2}$ indicates that an optimized value of $P_{\max }$ is reached for grid parameters considered for design, that is, $n$ and $w_{b}$. The above optimization process is repeated for desired range 


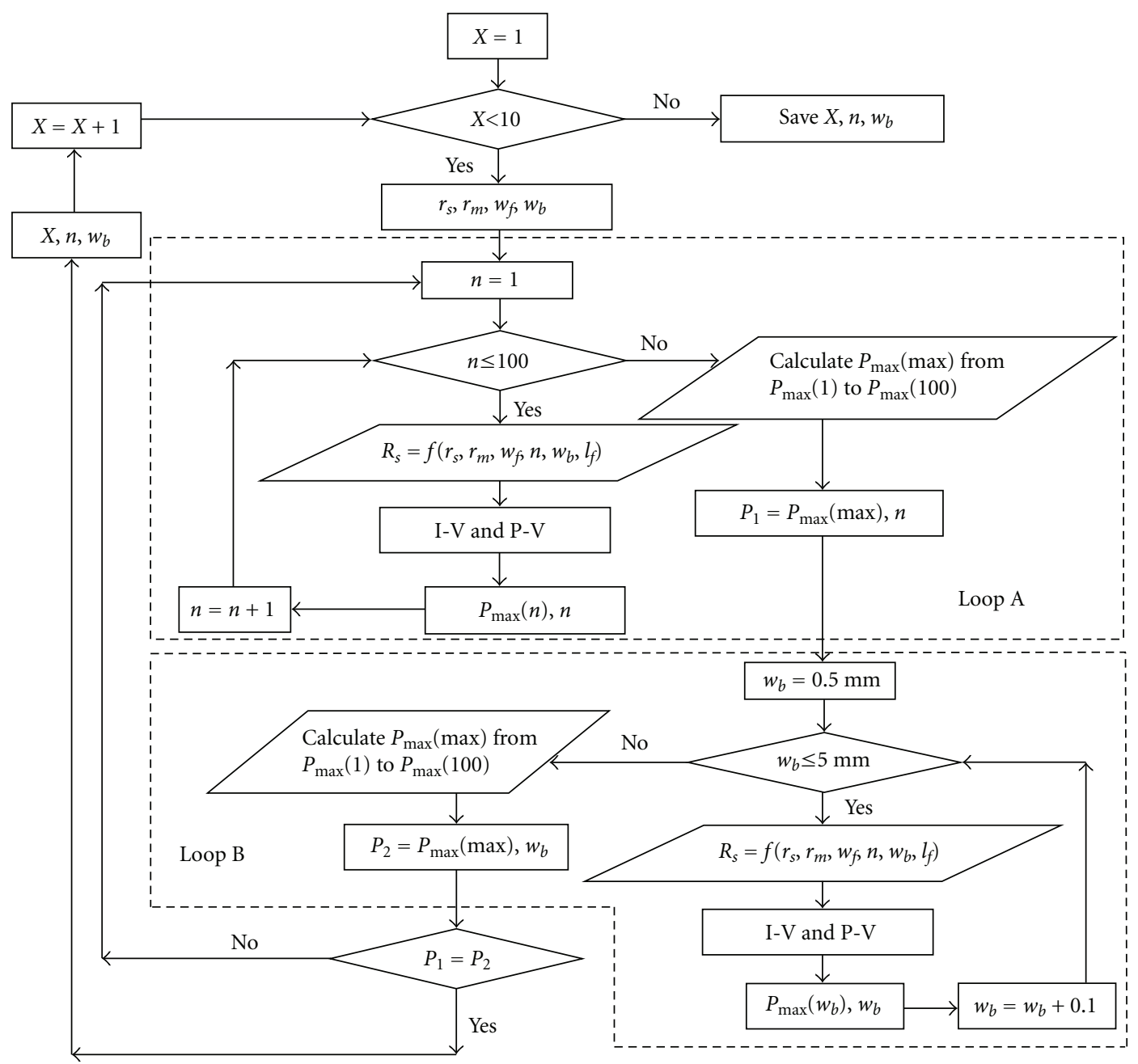

FIGURE 2: Flow chart for grid geometry optimization.

of concentration ratio. Thus for each concentration ratio the value of $n, w_{b}, P_{\max }$, as well as solar cell efficiency is obtained. The plot of normalized cell efficiencies (normalized with respect to 1 sun) of grid-optimized cells with respect to the concentration ratio is shown in Figure 4. The efficiencies of unoptimized 1 sun cell (case 0 ) as well as experimentally obtained efficiencies of a commercial 1 sun cell under light concentration are also plotted in Figure 4.

From Figure 4 it is clear that by optimizing the grid geometry (grid-optimized cell), the cell performance can be improved at the low concentration levels. The efficiency of the grid-optimized cell peaks at about 2-3 suns and at 10 suns, it drops only by about $10 \%$ of its value at 1 sun, whereas for unoptimized cell the efficiency reduces by about $30 \%$. As per the developed model, the resistive power loss at 10 suns is $20 \%$ and $42 \%$ of the generated power for the grid-optimized cell and unoptimized cell respectively. The reduction in efficiency of the grid-optimized cell, after it peaks at 2 suns is due to the increase in the resistive voltage drop as explained in (2). This fall in efficiency could be reduced further if the metallized area is increased by putting more number of fingers, but this would increase the shading and reduce the overall efficiency of the cell. Thus in order to keep the efficiency within limits the grid is optimized for 2 suns concentrations, and any further gird optimization for higher concentration reduces the efficiency of the cell.

The experimentally obtained efficiency of commercial 1 sun cell follows similar pattern with respect to concentration level as that of unoptimized cell but shows higher performance over range of concentration ratio. Such higher performance can be attributed to the fact that the commercial cell used for comparison was $4 \times 4 \mathrm{~cm}^{2}$ in size obtained by dicing from $15 \times 15 \mathrm{~cm}^{2}$ large area cells. The grids of the large area 1 sun commercial cells are designed to carry higher current than what is produced in $4 \times 4 \mathrm{~cm}^{2}$ area. Since current generated in $4 \times 4 \mathrm{~cm}^{2}$ cells is smaller, the resistive power losses in cells are smaller and hence performance is higher under light concentration. Normally the performance of small cells obtained from large area commercial cells peaks at about 2 to 3 suns concentration [18]. At concentration ratio higher than 3 suns, the commercial cell performance also drops steeply similar to the case of unoptimized cells due to limitation arising from resistive power losses. With these arguments we can say that the resistive model 


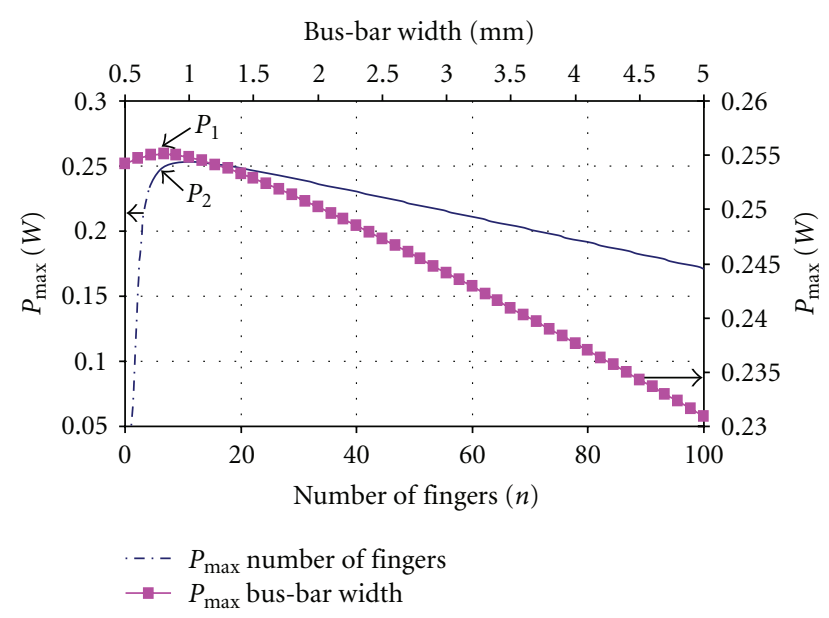

Figure 3: Optimization of $P_{\max }$ from iterating number of fingers and bus-bar width.

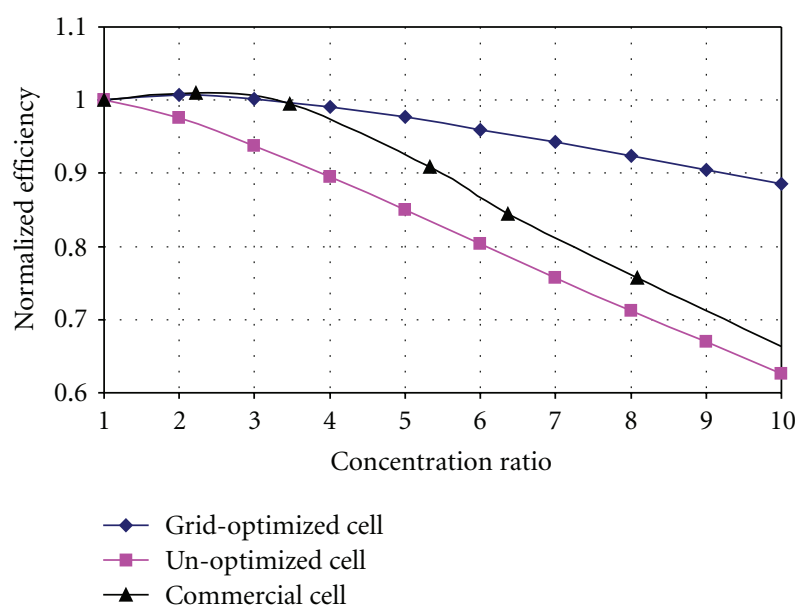

FIGURE 4: Normalized plot of Efficiency against the concentration ratio of the optimized and unoptimized grid solar cell.

developed for concentrator solar cells (Section 2) describes the unoptimized and grid-optimized cell reasonably well and can appropriately be used to evaluate the cell performance under low concentration levels.

From the above discussion it is explained that the gridoptimized cell is designed to have lower value of series resistance as compared to the commercial one sun cell and the unoptimized cell, but its series resistance is still higher for its operation at higher concentration levels of more than 2 suns. To overcome these limitations, junction depth optimization and electroplating of the grid contacts, an approach in designing of solar cells is proposed in the next sections.

\section{Case-2: Optimization of Junction Depth for Low-Concentrator Solar Cells}

The cell performance under low concentration levels can further be improved by emitter optimization, as stated earlier. The typical value of the emitter sheet resistance of a commercial Si solar cell is in the range of 40 to $60 \Omega$ /square. Recent studies have shown better results with the shallow emitter junction whose sheet resistance was as high as $95 \Omega$ /square [27]. Ideally the junction is required to be near the top surface (shallow junction) of the solar cells from where the light enters into the cell. The top surface generates a large quantity of electrons from the absorption of short wavelength light. The shallow junction result in higher value of sheet resistance as well as higher value of $J_{\mathrm{sc}}$, both of these results in higher resistive losses. This is not desired in the case of concentrator solar cell wherein the series resistance should be low. In order to reduce the losses in the emitter region, junction depth needs to be optimized such that it collects most of the generated electrons while providing low resistance path to current flow. This section explains the design methodology for junction depth optimization of a cell working under low concentration ratio.

Based on the study done in Section 3 for grid optimization, an algorithm is developed that, for a given concentration ratio, finds out an optimal junction depth and then the front grid is optimized to minimize overall series resistance of the cell, including that of emitter and grid. The flow chart for the optimization is shown in Figure 5. The algorithm is similar to gird geometry optimization as given in Figure 2. The only change incorporated in this case is that instead of using fixed value of sheet resistance we are calculating sheet resistance, $r_{s}$, depending on the junction depth, $x_{j}$, and the emitter resistivity, $\rho_{e}(5)$. The resistivity of the emitter layer is assumed fixed, with value of $8.85 \times$ $10^{-6} \Omega \mathrm{m}$ which corresponds to the emitter doping density of $N_{d}=1 \times 10^{20} \mathrm{~cm}^{-3}$ :

$$
r_{s}=\frac{\rho_{e}}{x_{j}}
$$

In this algorithm (Figure 5), the maximized peak power, $P_{3}$, corresponding to the optimum junction depth, $x_{j}$, is calculated using similar procedure as explained for the grid geometry parameters. This power is then compared with the $P_{1}$ and $P_{2}$ for their equality. The loop terminates when condition of $P_{1}=P_{2}=P_{3}$ is obtained. The condition of $P_{1}=P_{2}=P_{3}$ indicates that an optimized value of $P_{\max }$ is reached for grid parameters considered for design, that is $n$ and $w_{b}, x_{j}$. This search procedure is carried out for each concentration ratio under study.

Also a correlation for $J_{\mathrm{sc}}$ (used for peak power and resistive power loss estimation) as a function of junction depth, $x_{j}$, is written (refer to (6)) using the emitter doping profile. This corelation is obtained by solving the one dimensional diode equation under the illuminated condition.

$$
J_{\mathrm{sc}}=0.035 \times \exp ^{-0.5 x_{j}} .
$$

A plot of normalized efficiency with respect to the highest cell efficiency (shallow junction cell with $0.05 \mu \mathrm{m}$ junction depth) against the concentration ratio for different junction depthes is shown in Figure 6. It is observed that the shallow junction of about $0.05 \mu \mathrm{m}$ gives a better performance at low concentration range, upto 4 suns, as compared to the 


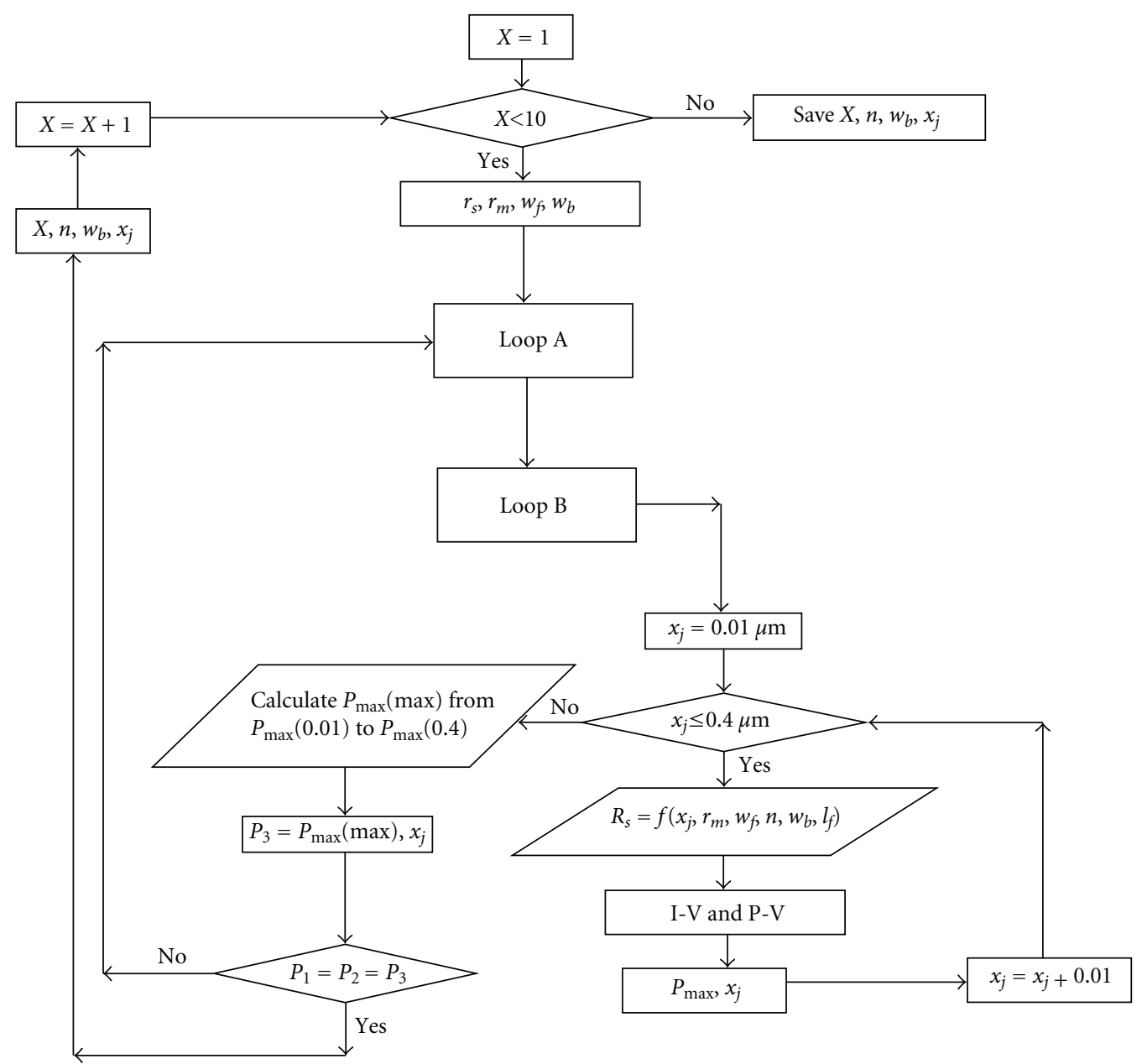

FIGURE 5: Flow chart for emitter junction depth optimization along with grid optimization.

cells with deeper junction depth, whereas there is drop in efficiency of $0.05 \mu \mathrm{m}$ cell at higher concentration ratio of 10 suns as against the deeper junction cells of 0.1 or $0.2 \mu \mathrm{m}$. The drop in the efficiency of a cell with $0.05 \mu \mathrm{m}$ junction depth at 10 suns concentration is due to higher resistive losses resulting from higher value of sheet resistance, $r_{s}$, and the higher value of current density generated (refer to (6)). In the mid concentration ranges of 4 suns to 7 suns the performance of cell with $0.1 \mu \mathrm{m}$ junction depth is better and beyond 7 suns; the performance of $0.15 \mu \mathrm{m}$ junction depth gives better results as compared to the shallow junction of $0.05 \mu \mathrm{m}$. Thus it could be concluded that in order for a commercial solar cell to operate at higher concentration levels junction depth should go on increasing in step by step sequence in order to reduce the overall series resistance. For the solar cell designed to work under 10 suns concentration ratio, the junction depth of $0.1 \mu \mathrm{m}$ should be chosen.

A normalized efficiency plot of junction-optimized cell, grid-optimized cell, un-optmized cell, and commercial 1 suns cell against the concentration ratio is shown in Figure 7. The plot also has information on junctionoptimized-electroplated cell and electroplated cell; discussion regarding these cell designs will be explained in later

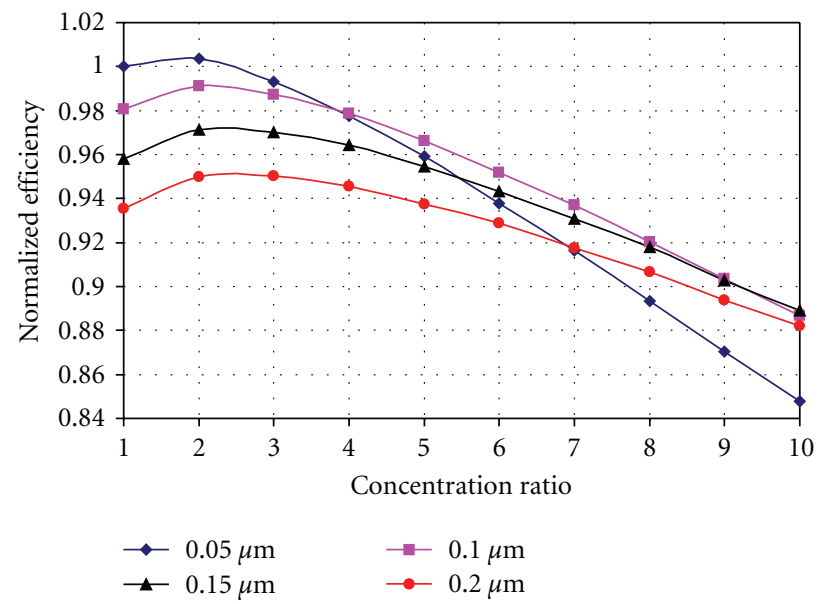

FIGURE 6: Normalized plot of the Junction depth-optimized solar cells (plot normalized with the highest efficiency).

section. For the present discussion the last four designs shown in the plot are considered. 


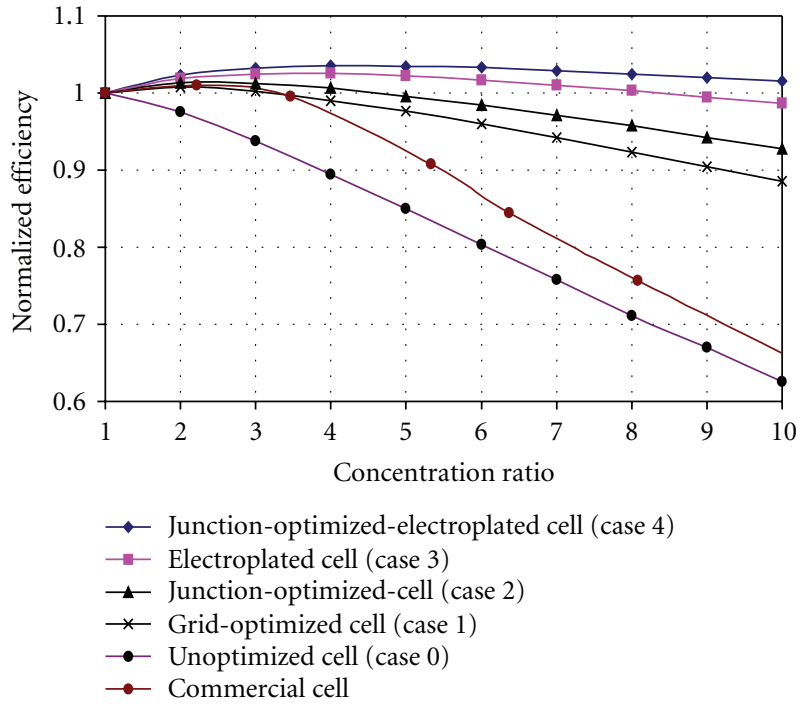

FIGURE 7: Normalized efficiency plot of cell designs for the junctionoptimized-electroplated cell, electroplated cell, grid-optimized cell, and unoptimized and commercial 1 sun cell.

Figure 7 shows improved performance of the junctionoptimized cell against the grid-optimized cell, unoptimized cell, and the commercial 1 sun cell at concentration levels of $2-10$ suns. An efficiency reduction of about 5\% for junctionoptimized cell is obtained at 10 suns as compared to the $10 \%$ reduction for grid-optimized cell, $25 \%$ reduction for commercial 1 sun, and 30\% reduction for unoptimized cell.

The resistive power loss at 10 suns is reduced from $20 \%$ for grid-optimized cell to about $16 \%$ for junctionoptimized cells. Thus by optimizing the junction depth the resistive power loss in the sheet resistance is reduced; a better performing solar cell at low concentration ratio can be designed.

\section{Case-3: Electroplated Front Grid Contacts on Solar Cells}

Front metal grid (fingers and bus-bars) and back Al metal contacts on solar cells are usually fabricated using screen printing of the metal paste. The front contact paste is a mixture of Ag with various organic bonders and additives while the back contact is $\mathrm{Al}$ paste. The screen printing paste has organic bonders and additives to make a better contact with Si. These bonders and additives evaporate during the cofiring of the contacts thus leaving behind the vacant spaces which result in reduced metal density of the fingers. This increases the sheet resistance and results in higher resistive power loss. Typically the sheet resistance of the screen printed contacts is in the range of 5 to $2 \mathrm{~m} \Omega$ /square for finger thickness of $25 \mu \mathrm{m}$ [28]. This value of sheet resistance is quite high for concentrator solar cell applications.

Once the contact printing and firing has taken place, the front contacts could then be electroplated in an Ag bath using light-induced plating techniques as explained by Mette et al. in [29]. The electroplating fills up the vacant spaces on the metal lines and increases the metal density. Due to electroplating the sheet resistance of the fingers and bus-bars reduces to about less than $1 \mathrm{~m} \Omega$ /square [18]. Electroplating also increases the width and thickness of the line which is certainly a better option in reducing the series resistance as overall crossection area of the fingers increases. The electroplated metal contacts can also be obtained without making use of screen-printed contacts wherein electroless deposition of $\mathrm{Ni}$ on $\mathrm{Si}$ in the form of grid pattern followed by electroplating of $\mathrm{Cu}$ is used [30].

The grid optimization study, described in Section 3, is used here to design and optimize grid pattern for the solar cell with electroplated front contacts. An algorithm, similar to what described in Section 3 (Figure 2), is used for grid optimization. The entire optimization sequence remains similar except that the metal sheet resistance, $r_{m}$, of the front contacts, corresponding to electroplated contacts, ( $1 \mathrm{~m} \Omega /$ square) is considered.

A comparison of normalized efficiency against concentration ratio for electroplated contacts cell, junctionoptimized cell, grid-optimized cell, unoptimized, and commercial 1 sun cell is shown in Figure 7. It is observed from the plot that there is an improvement in performance of the electroplated cells (case 3 ) as compared to the other cell design studied earlier. This improvement is due to reduction in metal line resistance, which leads to lower resistive power loss at concentration levels. At 10 suns, reduction in efficiency of the cell with electroplated contact by only about $1 \%$ as compared to 1 sun cell efficiency is observed. While for the same condition there is reduction of $5 \%$ for junctionoptimized (case 2), 10\% reduction for grid-optimized (case 1), $25 \%$ reduction for the commercial 1 sun cell, and $30 \%$ for unoptimized cell (case 0) (see Figure 7). The resistive power loss at 10 suns in case of the electroplated cell is about $11 \%$ (as estimated by resistive model of Section 2) of generated power which is much better than unoptimized 1 sun cell where the resistive power loss is $42 \%$ of the generated power.

\section{Case-4: Junction Depth Optimization for Electroplated Grid Solar Cells}

As studied in Section 4, junction depth optimization gave improved performance of the solar cells at concentration levels. The performance of the solar cell was improved further using electroplating the grid contacts. The present section studies the use of electroplated contacts with junction depth optimization for concentrator solar cell applications.

The procedure for optimizing junction depth remains similar to what shown in flow chart of Figure 5, which explains junction depth optimization with nonelectroplated contacts. The only difference in the present case is the sheet resistance of the emitter, which is considered to be a function of junction depth $\left(x_{j}\right)$ as shown in Equation (5) and front metal contacts sheet resistance. The front metal contacts are assumed to be electroplated with Ag and hence a lower value of sheet resistance $(1 \mathrm{~m} \Omega /$ square $)$ is used in the algorithm. For this case, a plot of normalized efficiency with respect to 


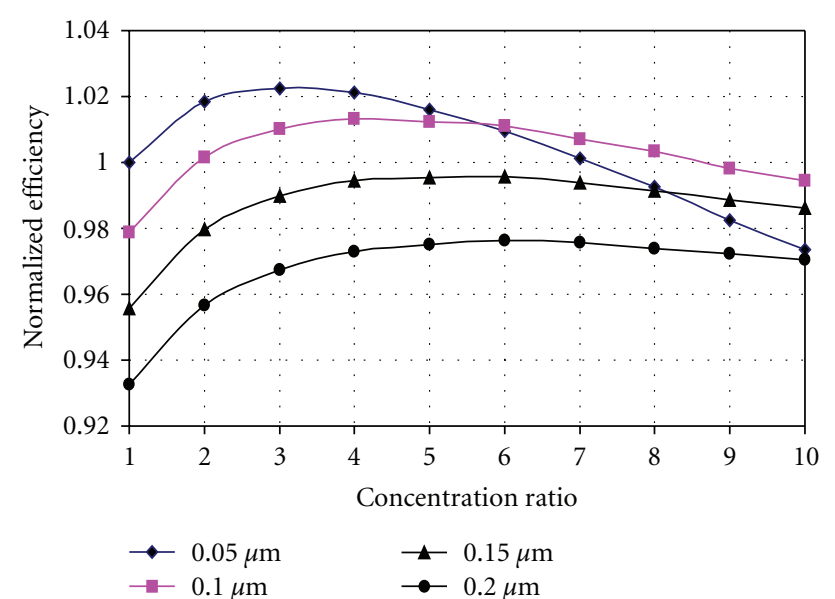

FIGURE 8: Normalized efficiency variation with concentration ratio for different junction depth on grid-optimized and electroplated solar cells.

the highest cell efficiency (shallow junction cell with $0.05 \mu \mathrm{m}$ junction depth) against the concentration ratio for different junction depth is shown in Figure 8. It is observed from the figure that the solar cell with shallow junction depth of about $0.05 \mu \mathrm{m}$ gives better performance at concentration levels of 2 to 5 suns. At higher concentration levels of more than 6 suns the performance of $0.05 \mu \mathrm{m}$ junction depth cell reduces and that of $0.1 \mu \mathrm{m}$ junction depth cell improves. This reduction in performance of $0.05 \mu \mathrm{m}$ cell is due to the increase in resistive power losses mostly due to sheet resistance at concentration levels of more than 5 suns. Thus a solar cell with junction depth of $0.1 \mu \mathrm{m}$ has a better performance as compared to the shallower $(0.05 \mu \mathrm{m})$ and deeper junction depth $(0.15$ and $0.2 \mu \mathrm{m})$ within the concentration ratios of 6 to 10 suns.

A normalized plot summarizing the results of all the cells designs (studied from Section 3 to Section 6) are shown in Figure 7. The plot shows how the performance of junctionoptimized-electroplated cell (case 4) is the best when compared with the previous cell designs at concentration ratios $2-10$ suns. The efficiencies obtained in case of junctionoptimized-electroplated cell are higher by about $2 \%$ to $3 \%$ at 10 suns than those obtained at 1 sun, while for other cell design there is a reduction in cell efficiency at 10 suns. Also the resistive power loss at 10 suns for junction-optimizedelectroplated cell is less than $10 \%$, which certainly indicates a reduction in the series resistance of the cell.

Table 2 shows the change in efficiency and the percentage resistive power loss at 10 suns for the various design of the solar cell studied.

It is shown that using the optimization techniques described in this paper for cell design, it is possible to design and fabricate low-concentrator c-Si solar cells (2- to 10 suns) using industrially viable cell processes. Among the four optimization processes described for concentrator solar cell design, the junction depth formation and front contact grid formation are the routine procedure followed during the solar cells fabrication. Which makes them suitable for
TABLe 2: Summary of results of cell design

\begin{tabular}{lcc}
\hline Cell design & $\begin{array}{c}\text { Approximate } \\
\text { change in } \\
\text { Efficiency at } \\
10 \text { suns }\end{array}$ & $\begin{array}{c}\text { Resistive Power } \\
\text { loss with respect to } \\
\text { power generated at } \\
10 \text { suns }\end{array}$ \\
\hline $\begin{array}{l}\text { Junction optimized, } \\
\text { electroplated cell } \\
\text { (case 4) }\end{array}$ & $+2-3 \%$ & $8.7 \%$ \\
$\begin{array}{l}\text { Electroplated cell } \\
\text { (case 3) }\end{array}$ & $-1 \%$ & $11.25 \%$ \\
$\begin{array}{l}\text { Junction optimized } \\
\text { cell (case 2) }\end{array}$ & $-5 \%$ & $16.49 \%$ \\
$\begin{array}{l}\text { Grid-optimized cell } \\
\text { (case 1) }\end{array}$ & $-10 \%$ & $20.15 \%$ \\
$\begin{array}{l}\text { Commercial 1 sun } \\
\text { (experimental data) }\end{array}$ & $-30 \%$ & Not available \\
$\begin{array}{l}\text { Unoptimized 1 sun } \\
\text { (case 0) }\end{array}$ & $-35 \%$ & $42.2 \%$ \\
\hline
\end{tabular}

implementation in case of low-concentrator cells without any additional process step. In case of electroplated contacts of case 3 an additional process of electroplating is required on the front metal contacts to reduce the series resistance. Since the electroplating is an industrial process which is carried out on a mass scale, it is possible to use this process on a commercial solar cell manufacturing. The use of light induce plating (LIP) has made the electroplating a much faster processing technique and hence a higher through put process.

\section{Conclusion}

A methodology for step by step reduction in series resistance for design of low level concentrator solar cell (2 to 10 suns) is presented. A resistive model is developed for analysis of concentrator solar cells. A commercial 1 sun solar cell is converted to work under low level concentration by optimizing the front grid and junction depth and varying the front metal lines resistance. These optimization processes can be implemented in commercial fabrication setup of solar cells. At 10 suns, the estimated resistive power loss as compared to generated power is $42 \%, 20 \%, 16 \%, 11 \%$ and $8 \%$ for unoptimized cell, grid-optimized cell, junctionoptimized cell, electroplated cell, and junction-optimized, electroplated cell. The reducing trend of resistive power loss indicates that the proposed optimized commercial cell can be used for increasing concentration ratio up to 10 suns. A commercial cell optimized for grid junction and low metal line resistance has shown an efficiency improvement of 3\% at 10 suns concentration.

\section{Appendix}

The calculation of series resistance from the individual resistance is explained in the appendix. Power loss equations in emitter grid are used to calculate the resistance in that region. Continuity equations are used in deriving the power 


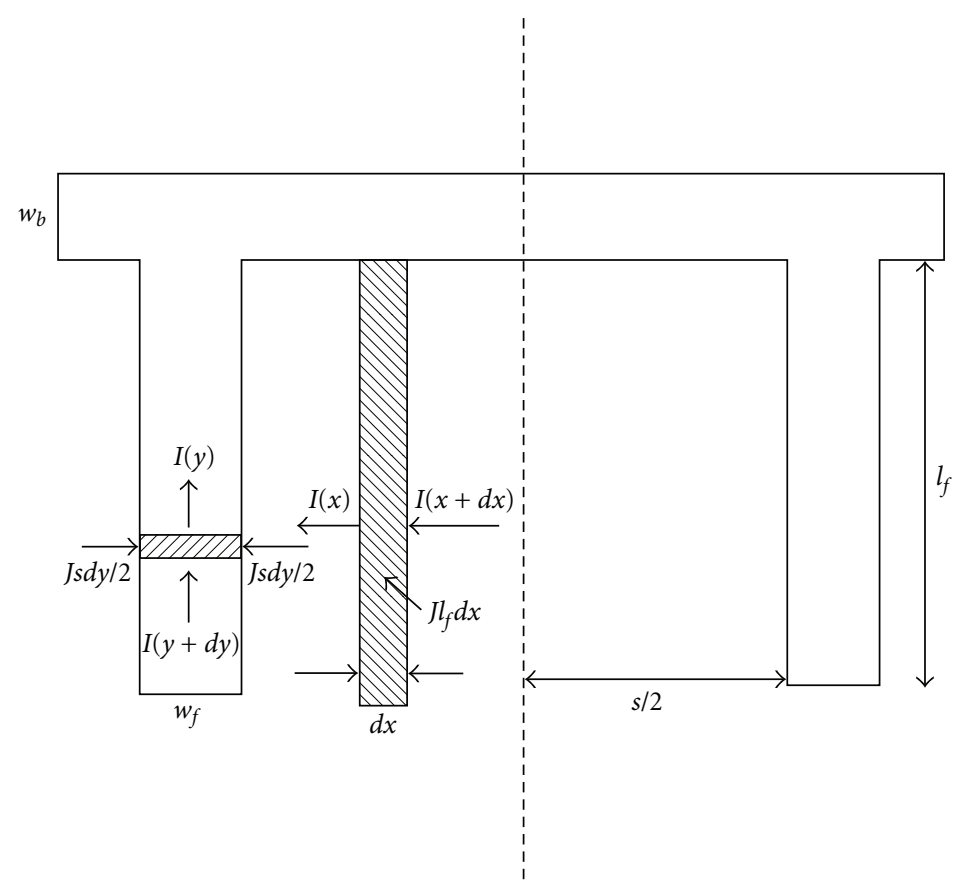

Figure 9: Section of the top region of the solar cell indicating the fingers, bus-bars, and the emitter region.

loss equations. Figure 9 shows a top section of a solar cell showing a section of fingers, bus-bar, and the emitter. The current generated in the emitter is collected in the fingers and then transported to the bus bars. The current flow follows a continuity equation given as

$$
d I(x)=-J_{s c} l_{f} d x,
$$

where $l_{f}$ is the length of the fingers and the $J_{\mathrm{sc}}$ is the current density.

Integrating (A.1) with boundary conditions $x=s / 2$, $I(0)=0$ we have

$$
I(x)=J_{s c} l_{f}\left(\frac{s}{2}-x\right) .
$$

The power loss in the emitter region is given as

$$
d P_{e}=I(x) * d R_{e},
$$

where $d R_{e}$ is the emitter resistance and is given as

$$
d R_{e}=\frac{r_{s} d x}{l_{f}} .
$$

$r_{s}$ is the sheet resistance of the emitter layer.

The power loss from (A.2) and (A.4) is given as

$$
d P_{e}=J_{\mathrm{sc}}{ }^{2} l_{f}{ }^{2}\left(\frac{s}{2}-x\right)^{2} \frac{r_{s} d x}{l_{f}} .
$$

Integrating (A.5) within limits $s / 2$ to 0 we obtain the power loss in the emitter region:

$$
P_{e}=J_{\mathrm{sc}}^{2} l_{f}^{2} s^{2} \frac{s r_{s}}{24 l_{f}} .
$$

Separating the power loss equation as $I^{2} R_{e}$ we obtain the emitter resistance in a small region of area $\left(s^{*} l_{f}\right) / 2$ and we get

$$
R_{e}=\frac{s r_{s}}{24 l_{f}} .
$$

A similar equation is derived for the power loss in a finger and a corresponding resistance in a finger is calculated as

$$
R_{f}=\frac{r_{m} l_{f}}{3 w_{f}} .
$$

The resistance of the bus-bar is assumed distributed, and its value $R_{\mathrm{bus}}$ between the two corresponding fingers is considered (shown in Figure 10). This value is then used in the ladder-network and solved to obtain the overall series resistance of the solar cell:

$$
R_{\mathrm{bus}}=\frac{r_{m}\left(s+w_{f}\right)}{w_{b}} .
$$

The remaining resistance is the contact resistance $R_{f c}$ between the front contact grid and the emitter, calculated from the specific contact resistance $\sigma_{c}$ and the contact area of the finger; the contact resistance due to the bus-bar is neglected due to its larger contact area:

$$
R_{f c}=\frac{\sigma_{c}}{w_{f} l_{f}} .
$$

The bulk resistance is calculated from the bulk resistivity, thickness and the area of the solar cell,

$$
R_{\text {bulk }}=\frac{\rho_{\text {bulk }} t}{A_{c}} .
$$




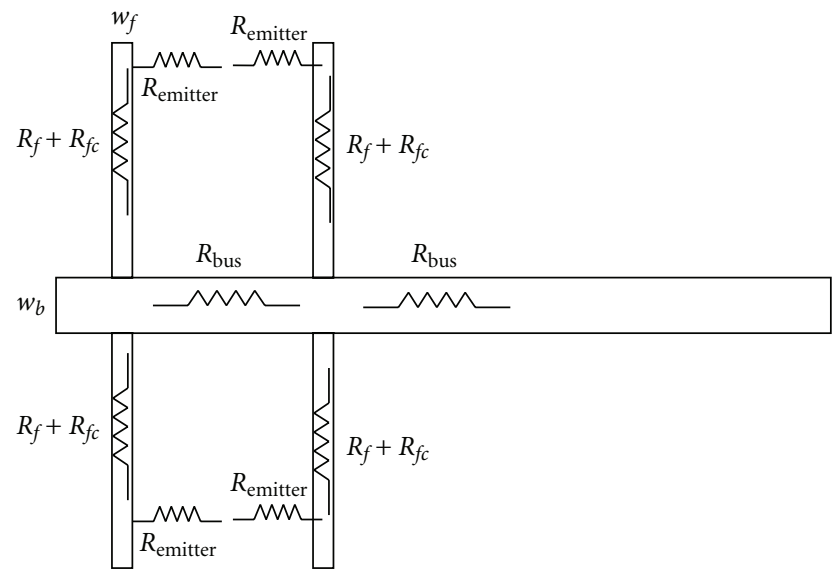

FIGURE 10: Indicating the resistive network in the top region of the solar cell.

The contact resistance due to the back contact is neglected due to the large back contact area which is the area of the solar cell.

The overall series resistance is given by the following equation:

$$
R_{s}=R_{\mathrm{bus}}+R_{\mathrm{bulk}}+\frac{R_{\mathrm{emitter}}}{2(n+1)}+f\left(n,\left(R_{f}+R_{f c}\right), R_{\mathrm{bus}}\right) .
$$

\section{References}

[1] http://www.solarbuzz.com/Marketbuzz2009-intro.htm, February 2009.

[2] H. A. Aulich and F.-W. Schulze, "Crystalline silicon feedstock for solar cells," Progress in Photovoltaics: Research and Applications, vol. 10, no. 2, pp. 141-147, 2002.

[3] W. Hoffmann, "PV solar electricity industry: market growth and perspective," Solar Energy Materials and Solar Cells, vol. 90, no. 18-19, pp. 3285-3311, 2006.

[4] http://www.solarbuzz.com, February 2009.

[5] L. L. Kazmerski, "Solar photovoltaics R\&D at the tipping point: a 2005 technology overview," Journal of Electron Spectroscopy and Related Phenomena, vol. 150, no. 2-3, pp. 105-135, 2006.

[6] K. J. Weber, A. W. Blakers, P. N. K. Deenapanray, V. Everett, and E. Franklin, "Sliver® solar cells," in Proceedings of the 31st IEEE Photovoltaic Specialists Conference, pp. 991-994, IEEE, Orlando, Fla, USA, January 2005.

[7] R. Mertens, "Trends in solar cell research," in Proceedings of the 15th International Symposium on the Physical and Failure Analysis of Integrated Circuits (IPFA '08), pp. 1-5, July 2008.

[8] I. A. Schwirtlich, "EFG ribbon technology," in High-Efficient Low-Cost Photovoltaics, vol. 140 of Springer Series in Optical Sciences, pp. 57-64, Springer, Berlin,Germany, 2009.

[9] B. Pivac, V. Borjanovi, I. Kovacevi, B. N. Evtody, and E. A. Katz, "Comparative studies of EFG poly-Si grown by different procedures," Solar Energy Materials and Solar Cells, vol. 72, no. 1-4, pp. 165-171, 2002.

[10] M. A. Green, P. A. Basore, N. Chang, et al., "Crystalline silicon on glass (CSG) thin-film solar cell modules," Solar Energy, vol. 77, no. 6, pp. 857-863, 2004.
[11] P. A. Basore, "CSG-1: manufacturing a new polycrystalline silicon PV technology," in Proceedings of the 4thIEEE World Conference on Photovoltaic Energy Conversion (WCPEC '07), vol. 2, pp. 2089-2093, May 2007.

[12] A. Cuevas, R. A. Sinton, N. E. Midkiff, and R. M. Swanson, "26-percent efficient point-junction concentrator solar cells with a front metal grid," Electron Device Letters, vol. 11, no. 1, pp. 6-8, 1990.

[13] F. Zhang, S. Wenham, and M. A. Green, "Large area, concentrator buried contact solar cells," IEEE Transactions on Electron Devices, vol. 42, no. 1, pp. 144-149, 1995.

[14] M. Garcia, L. Marroyo, E. Lorenzo, and M. Perez, "Experimental energy yield in 1.5X and 2X PV concentrators with conventional modules," Progress in Photovoltaics: Research and Applications, vol. 16, pp. 261-270, 2008.

[15] C. S. Sangani and C. S. Solanki, "Experimental evaluation of V-trough (2 suns) PV concentrator system using commercial PV modules," Solar Energy Materials and Solar Cells, vol. 91, no. 6, pp. 453-459, 2007.

[16] Z. I. Alferov, V. M. Andreev, and V. D. Rumyantsev, "III-V solar cells and concentrator arrays," in High-Efficient Low-Cost Photovoltaics, vol. 140 of Springer Series in Optical Sciences, pp. 101-141, Springer, Berlin, Germany, 2009.

[17] D. C. Law, R. R. King, H. Yoon, et al., "Future technology pathways of terrestrial III-V multijunction solar cells for concentrator photovoltaic systems," Solar Energy Materials and Solar Cells. In press.

[18] J. Coello, M. Castro, I. Antón, G. Sala, and M. A. Vázquez, "Conversion of commercial Si solar cells to keep their efficient performance at 15 suns," Progress in Photovoltaics: Research and Applications, vol. 12, no. 5, pp. 323-331, 2004.

[19] M. Castro, I. Anton, and G. Sala, "Pilot production of concentrator silicon solar cells: approaching industrialization," Solar Energy Materials and Solar Cells, vol. 92, no. 12, pp. 16971705, 2008.

[20] I. Luque-Heredia, C. Martín, M. T. Mañanes, et al., "A subdegree precision sun tracker for $1000 \mathrm{X}$ microconcentrator modules," in Proceddings of the 3rd World Conference on Photovoltaic Energy Conversion, pp. 857-860, Osaka, Japan, 2003.

[21] A. Luque, Solar Cells and Optics for Photovoltaic Concentration, chapter 4, Adam Hilger, Bristol, UK, 1990.

[22] A. Antonini, M. Stefancich, D. Vincenzi, et al., "Contact grid optimization methodology for front contact concentration solar cells," Solar Energy Materials and Solar Cells, vol. 80, no. 2, pp. 155-166, 2003.

[23] D. Pysch, A. Mette, and S. W. Glunz, "A review and comparison of different methods to determine the series resistance of solar cells," Solar Energy Materials and Solar Cells, vol. 91, no. 18, pp. 1698-1706, 2007.

[24] P. N. Vinod, "Evaluation of the ohmic properties of the silver metal contacts of an improved sintering process on the multicrystalline silicon solar cells," in Proceedings of the 14th International Workshop on the Physics of Semiconductor Devices (IWPSD '07), pp. 953-956, Bombay, India, 2007.

[25] R. J. Handy, "Theoretical analysis of the series resistance of a solar cell," Solid State Electronics, vol. 10, no. 8, pp. 765-775, 1967.

[26] M. Green, Solar Cells, Prentice-Hall, Englewood Cliffs, NJ, USA, 1987.

[27] V. Yelundur, K. Nakayashiki, M. Hilali, and A. Rohatgi, "Implementation of a homogeneous high-sheet-resistance emitter in multicrystalline silicon solar cells," in Proceedings of IEEE Photovoltaic Specialists Conference, pp. 959-962, 2005. 
[28] Data Sheet from Ferro Corporation, NS 33-502 Front Contact Silver for SiN Passivated Solar Cell.

[29] A. Mette, C. Schetter, D. Wissen, S. Lust, S. W. Glunz, and G. Willeke, "Increasing the efficiency of screen-printed silicon solar cells by light-induced silver plating," in Proceedings of the 4th IEEE World Conference on Photovoltaic Energy Conversion (WCPEC '07), vol. 1, pp. 1056-1059, May 2007.

[30] J. A. D. Jensen, P. Møller, T. Bruton, et al., "Electrochemical deposition of buried contacts in high-efficiency crystalline silicon photovoltaic cells," Journal of the Electrochemical Society, vol. 150, no. 1, pp. G49-G57, 2003. 


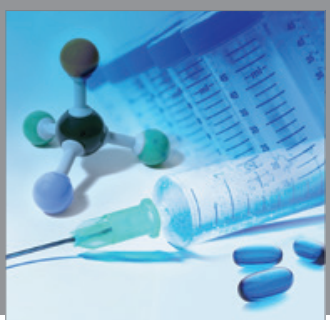

International Journal of

Medicinal Chemistry

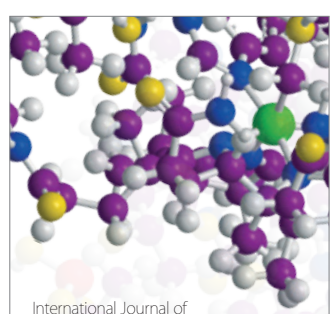

Carbohydrate Chemistry

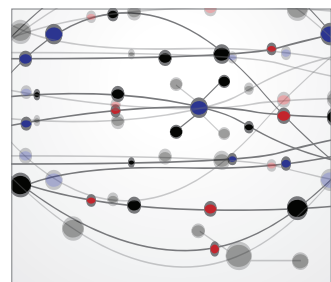

The Scientific World Journal
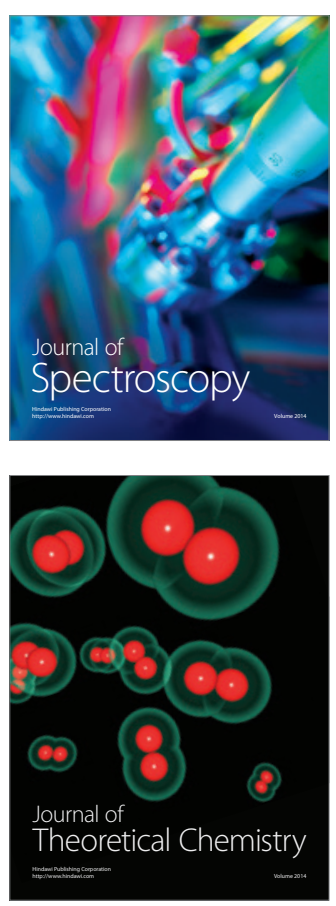
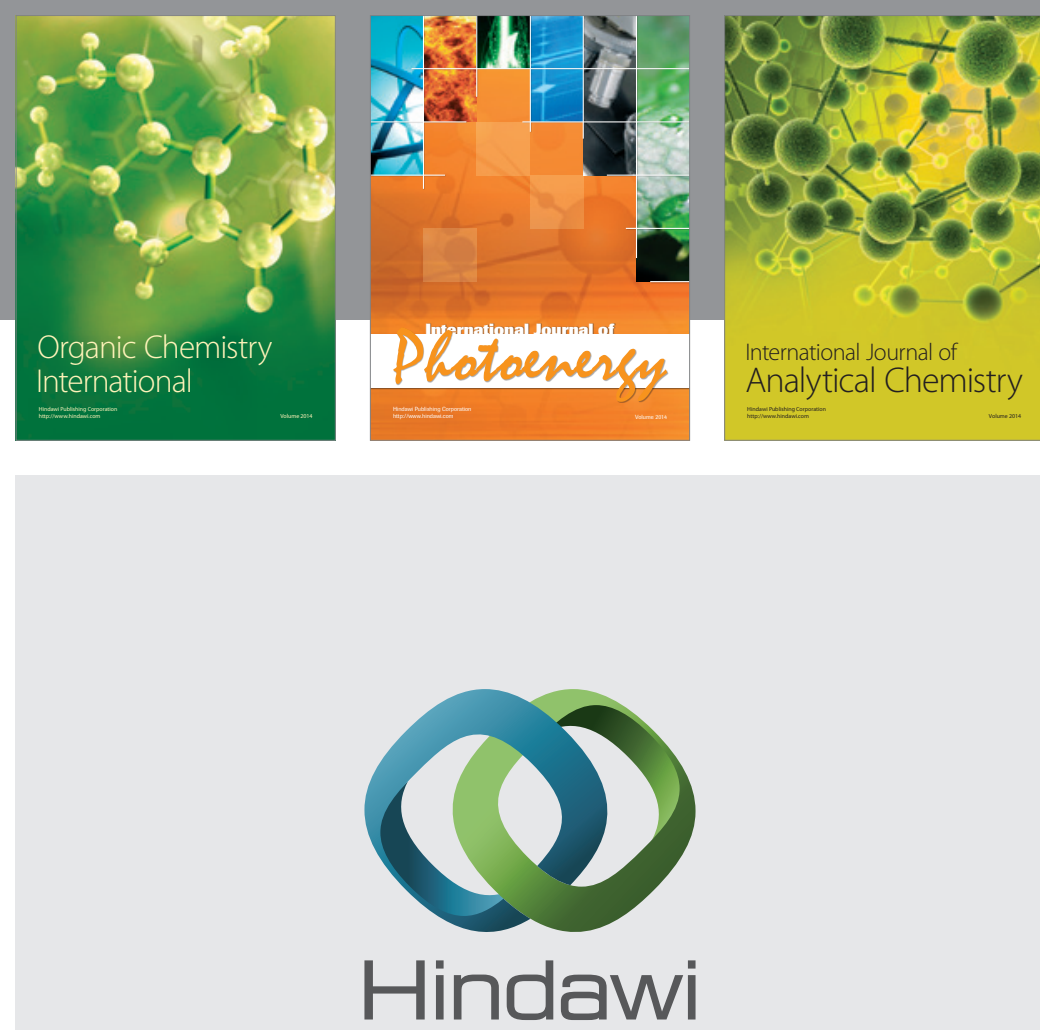

Submit your manuscripts at

http://www.hindawi.com
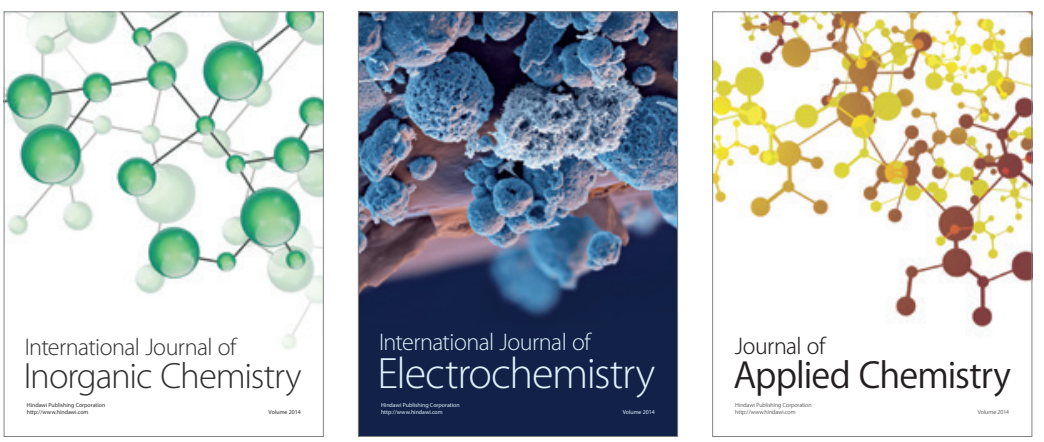

Journal of

Applied Chemistry
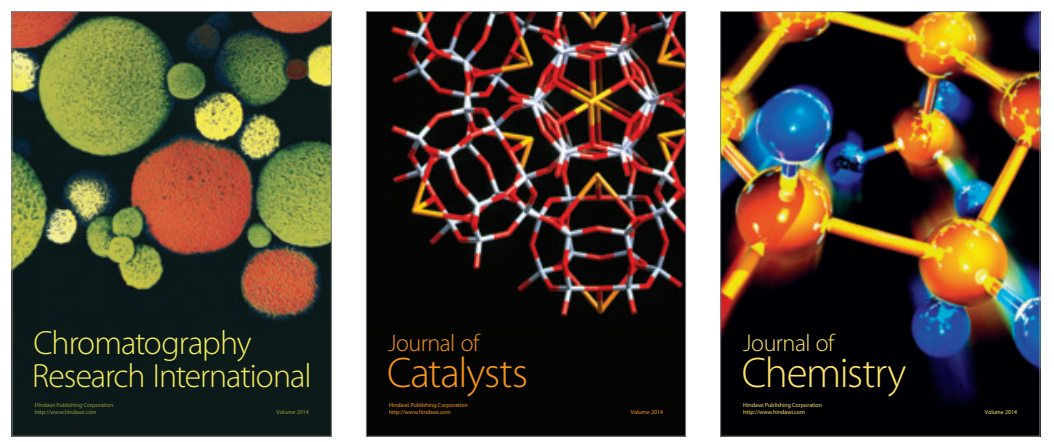
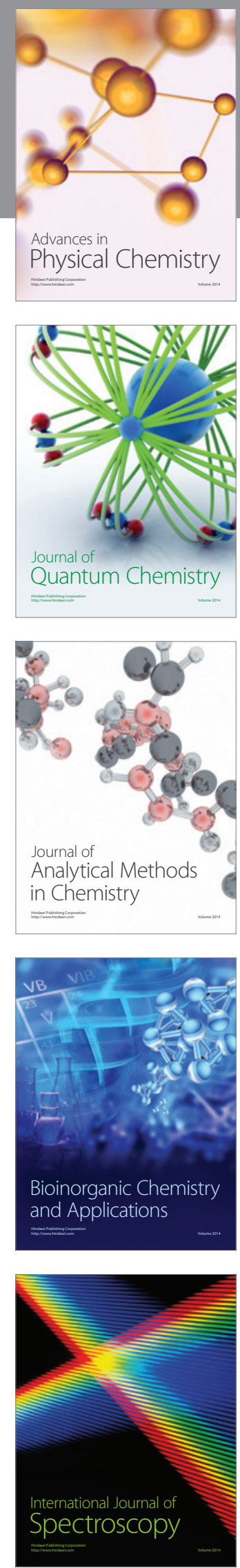